\title{
CHARACTERIZATION OF METRIC SPACES WHOSE FREE SPACE IS ISOMETRIC TO $\ell_{1}$
}

\author{
AUDE DALET $^{\dagger}$, PEDRO L. KAUFMANN ${ }^{\ddagger}$, AND ANTONÍN PROCHÁZKA ${ }^{\dagger}$
}

\begin{abstract}
We characterize metric spaces whose Lipschitz free space is isometric to $\ell_{1}$. In particular, the Lipschitz free space over an ultrametric space is not isometric to $\ell_{1}(\Gamma)$ for any set $\Gamma$. We give a lower bound for the Banach-Mazur distance in the finite case.
\end{abstract}

\section{INTRODUCTION}

An $\mathbb{R}$-tree $(T, d)$ is a metric space which is geodesic and satisfies the 4-point condition:

$$
\forall a, b, c, d \in T \quad d(a, b)+d(c, d) \leq \max \{d(a, c)+d(b, d), d(b, c)+d(a, d)\} .
$$

A space which satisfies just the 4-point condition is called 0-hyperbolic. Clearly, a subset of an $\mathbb{R}$-tree is 0 -hyperbolic. The converse is also true [4, 7], so we will use terms " 0 -hyperbolic" and "subset of an $\mathbb{R}$-tree" interchangeably. Moreover, for every 0-hyperbolic $M$ there exists a unique (up to isometry) minimal $\mathbb{R}$-tree which contains $M$, we will denote it $\operatorname{conv}(M)$. Thus one can define the Lebesgue measure $\lambda(M)$ of $M$ which is independent of any particular tree containing $M$. We will say that $M$ is negligible if $\lambda(M)=0$. A. Godard [9] has proved that a metric space $M$ is 0-hyperbolic if and only if $\mathcal{F}(M)$ is isometric to a subspace of some $L_{1}(\mu)$. In this paper we are interested in metric spaces whose free space is isometric to (a subspace of) $\ell_{1}$. By the above, such spaces must be 0-hyperbolic, and it is also easy to see that they must be negligible (if not the free space will contain $L_{1}$ ).

So let $M$ be a separable negligible complete metric space which is a subset of an $\mathbb{R}$-tree. One can ask two questions:

- When is $\mathcal{F}(M)$ isometric to $\ell_{1}$ ?

- When is $\mathcal{F}(M)$ isometric to a subspace of $\ell_{1}$ ?

Concerning the first question, the results of A. Godard point to the relevance of branching points of $\operatorname{conv}(M)$. We recall that a point $b \in T$ is a branching point of a tree $T$ if $T \backslash\{b\}$ has at least three connected components. A sufficient condition for $\mathcal{F}(M) \equiv \ell_{1}$ is that $M$ contain all the branching points of $\operatorname{conv}(M)$ [9, Corollary 3.4]. The main result of this paper (Theorem 5) claims that this is also a necessary condition. We give two different proofs - one is based on properties of the extreme points of $B_{\mathcal{F}(M)}$ and the other on properties of the extreme points of $B_{\text {Lip }_{0}(M)}$ (Theorem 4).

For certain finite 0 -hyperbolic spaces $M$ we have a third proof which also allows to compute a simple lower bound for the Banach-Mazur distance between $\mathcal{F}(M)$ and $\ell_{1}^{|M|-1}$ (Proposition 9).

As far as the second question is concerned, it is obviously enough that $M$ be a subset of a metric space $N$ such that $\mathcal{F}(N) \equiv \ell_{1}$. We will show that this is the case when $M$ is compact, 0-hyperbolic and negligible (Proposition 8). We do not know whether one can drop the assumption of compactness in general. 
This paper is an outgrowth of a shorter preprint in which we have shown that for any ultrametric space $M$, the free space $\mathcal{F}(M)$ is never isometric to $\ell_{1}$ (Corollary 6) answering a question posed by M. Cúth and M. Doucha in a draft of [5]. In the meantime, this question has been independetly answered in [5].

\section{Preliminaries}

As usual, for a metric space $M$ with a distinguished point $0 \in M$, the Lipschitz-free space $\mathcal{F}(M)$ is the norm-closed linear span of $\left\{\delta_{x}: x \in M\right\}$ in the space $\operatorname{Lip}_{0}(M)^{*}$, where the Banach space $\operatorname{Lip}_{0}(M)=\left\{f \in \mathbb{R}^{M}: f\right.$ Lipschitz, $\left.f(0)=0\right\}$ is equipped with the norm $\|f\|_{L}:=\sup \left\{\frac{f(x)-f(y)}{d(x, y)}: x \neq y\right\}$. It is well known that $\mathcal{F}(M)^{*}=\operatorname{Lip}_{0}(M)$ isometrically. More about the very interesting class of Lipschitz-free spaces can be found in [10].

To prove a Lispchitz-free space is not isometric to $\ell_{1}$, we will exhibit two extreme points of its unit ball at distance less than one. For this purpose we will use the notion of peaking function at $(x, y), x \neq y$, which is a function $f \in \operatorname{Lip}_{0}(M)$ such that $\frac{f(x)-f(y)}{d(x, y)}=1$ and for every open set $U$ of $\{(x, y) \in M \times M, x \neq y\}$ containing $(x, y)$ and $(y, x)$, there exists $\delta>0$ with $(z, t) \notin U \Rightarrow \frac{|f(z)-f(t)|}{d(z, t)} \leq 1-\delta$. This definition is equivalent to: $\frac{f(x)-f(y)}{d(x, y)}=1$ and if $\left(u_{n}\right)_{n \in \mathbb{N}},\left(u_{n}\right)_{n \in \mathbb{N}} \subset M$, then

$$
\lim _{n \rightarrow+\infty} \frac{f\left(u_{n}\right)-f\left(v_{n}\right)}{d\left(u_{n}, v_{n}\right)}=1 \Rightarrow \lim _{n \rightarrow+\infty} u_{n}=x \text { and } \lim _{n \rightarrow+\infty} v_{n}=y
$$

Moreover in [11, Proposition 2.4.2], the following is proved:

Proposition 1. Let $(M, d)$ be a complete metric space and $x \neq y$ in $M$. If there is a function $f \in \operatorname{Lip}_{0}(M)$ peaking at $(x, y)$, then $\frac{\delta_{x}-\delta_{y}}{d(x, y)}$ is an extreme point of the unit ball of $\operatorname{Lip}_{0}(M)^{*}$. In particular, it is an extreme point of the unit ball of $\mathcal{F}(M)$.

Given an $\mathbb{R}$-tree $(T, d)$ and $x, y \in T$, the segment $[x, y]$ is defined as the range of the unique isometry $\phi_{x, y}$ from $[0, d(x, y)] \subset \mathbb{R}$ into $T$ which maps 0 to $x$ and $d(x, y)$ to $y$.

We recall that for every 0-hyperbolic space $M$, there exists an $\mathbb{R}$-tree $T$ such that $M \subset T$. The set $\bigcup\{[x, y]: x, y \in M\} \subset T$ is then also an $\mathbb{R}$-tree. It is clearly a minimal $\mathbb{R}$-tree containing $M$; it is unique up to an isometry and will be denoted conv $(M)$. Simple examples show that $\operatorname{conv}(M)$ does not have to be complete when $M$ is. This does not suppose any difficulty in what follows.

A point $b \in T$ is said to be a branching point if there are three distinct points $x, y, z \in T \backslash\{b\}$ with $[x, b] \cap[y, b]=[x, b] \cap[z, b]=[y, b] \cap[z, b]=\{b\}$. We say that the branching point $b$ is witnessed by $x, y, z$. The set of all branching points of $T$ is denoted $\operatorname{Br}(T)$. If $M$ is 0-hyperbolic, the set of all branching points of $\operatorname{conv}(M)$ is denoted $\operatorname{Br}(M)$.

A subset $A$ of $T$ is measurable if $\phi_{x, y}^{-1}(A)$ is Lebesgue-measurable, for every $x$ and $y$ in $T$. For a segment $S=[x, y]$ in $T$ and $A$ measurable, we denote $\lambda_{S}(A):=\lambda\left(\phi_{x, y}^{-1}(A)\right)$, with $\lambda$ the Lebesgue measure on $\mathbb{R}$. Let $\mathcal{R}$ be the set of subsets of $T$ that can be written as a finite 
union of disjoint segments. For $R=\bigcup_{k=1}^{r} S_{k} \in \mathcal{R}$, define $\lambda_{R}(A):=\sum_{k=1}^{r} \lambda_{S_{k}}(A)$ and finally, set $\lambda_{T}(A):=\sup _{R \in \mathcal{R}} \lambda_{R}(A)$. If $M$ is 0-hyperbolic, we put simply $\lambda(M):=\lambda_{\operatorname{conv}(M)}(M)$.

Given two points $x$ and $y$ in $T$, we will denote $\pi_{x y}: T \rightarrow[x, y]$ the metric projection onto the segment $[x, y]$. It is well known and easily seen that $\pi_{x y}$ is non-expansive (see $\left.[1,3]\right)$.

Finally, we recall that a metric space $(M, d)$ is ultrametric if $d(x, y) \leq \max \{d(x, z), d(y, z)\}$ for any $x, y, z \in M$.

\section{IsOmetries With $\ell_{1}$}

Let us start by characterizing precisely when there exists a function peaking at $(x, y)$ for points $x, y \in M \subset T$.

Proposition 2. Let $(M, d)$ be a complete subset of an $\mathbb{R}$-tree and $x, y \in M, x \neq y$. The following assertions are equivalent

(i) There is $f \in \operatorname{Lip}_{0}(M)$ peaking at $(x, y)$.

(ii) $M \cap[x, y]=\{x, y\}$ and for every $p \in\{x, y\}$,

$$
\left.\liminf _{u, v \rightarrow p} \frac{d\left(\pi_{x y}(u), u\right)+d\left(\pi_{x y}(v), v\right)}{d\left(\pi_{x y}(u), \pi_{x y}(v)\right)}>0, \text { (with the convention that } \frac{\alpha}{0}=+\infty\right) \text {. }
$$

(iii) $M \cap[x, y]=\{x, y\}$ and for every $p \in\{x, y\}$,

$$
\left.\liminf _{u \rightarrow p} \frac{d\left(\pi_{x y}(u), u\right)}{d\left(\pi_{x y}(u), p\right)}>0, \text { (with the convention that } \frac{\alpha}{0}=+\infty\right) \text {. }
$$

Proof. (ii) $\Rightarrow$ (i) Let us first suppose that $x, y$ satisfy (1) and $[x, y] \cap M=\{x, y\}$. For any $u \in M$ we define $f(u)=d\left(y, \pi_{x y}(u)\right)$. Then $\frac{f(x)-f(y)}{d(x, y)}=1$ and $\|f\|_{L}=1$. Consider $\left(x_{n}\right)_{n \in \mathbb{N}},\left(y_{n}\right)_{n \in \mathbb{N}} \subset M$ such that $\lim _{n \rightarrow+\infty} \frac{f\left(x_{n}\right)-f\left(y_{n}\right)}{d\left(x_{n}, y_{n}\right)}=1$. We thus have for $n$ large enough

$$
d\left(y, \pi_{x y}\left(x_{n}\right)\right)=f\left(x_{n}\right)>f\left(y_{n}\right)=d\left(y, \pi_{x y}\left(y_{n}\right)\right) .
$$

It follows

$$
1=\lim _{n \rightarrow+\infty} \frac{f\left(x_{n}\right)-f\left(y_{n}\right)}{d\left(x_{n}, y_{n}\right)}=\lim _{n \rightarrow+\infty} \frac{d\left(\pi_{x y}\left(x_{n}\right), \pi_{x y}\left(y_{n}\right)\right)}{d\left(x_{n}, \pi_{x y}\left(x_{n}\right)\right)+d\left(\pi_{x y}\left(x_{n}\right), \pi_{x y}\left(y_{n}\right)\right)+d\left(\pi_{x y}\left(y_{n}\right), y_{n}\right)}
$$

and in particular

$$
\lim _{n \rightarrow \infty} \frac{d\left(x_{n}, \pi_{x y}\left(x_{n}\right)\right)+d\left(\pi_{x y}\left(y_{n}\right), y_{n}\right)}{d\left(\pi_{x y}\left(x_{n}\right), \pi_{x y}\left(y_{n}\right)\right)}=0 .
$$

Since $\lim _{n \rightarrow+\infty} d\left(x_{n}, \pi_{x y}\left(x_{n}\right)\right)=\lim _{n \rightarrow+\infty} d\left(y_{n}, \pi_{x y}\left(y_{n}\right)\right)=0$, the sets of cluster points of the sequences $\left(\left(\pi_{x y}\left(x_{n}\right), \pi_{x y}\left(y_{n}\right)\right)\right)_{n \in \mathbb{N}} \subset[x, y]^{2}$ and $\left(\left(x_{n}, y_{n}\right)\right)_{n \in \mathbb{N}} \subset M^{2}$ coincide. By compactness of $[x, y]^{2}$ there exists such a cluster point $(u, v) \in[x, y]^{2}$. Since the space $M$ is complete, $(u, v) \in M^{2}$, and therefore $(u, v) \in\{(y, x),(x, x),(y, y),(x, y)\}$. Clearly, (3) implies $(u, v) \neq(y, x)$, and $(1)$ together with (4) imply that $(u, v) \neq(x, x)$ and $(u, v) \neq(y, y)$. We thus get that $\left(x_{n}\right)$ converges to $x$ and $\left(y_{n}\right)$ converges to $y$ which proves that $f$ is peaking at $(x, y)$. 
(i) $\Rightarrow$ (iii) If there is $z \in M \cap(x, y)$, then $\frac{\delta_{x}-\delta_{y}}{d(x, y)}$ is a convex combination of $\frac{\delta_{x}-\delta_{z}}{d(x, z)}$ and $\frac{\delta_{z}-\delta_{y}}{d(z, y)}$ so by Proposition 1, there cannot be a peaking function at $(x, y)$.

Next assume that $[x, y] \cap M=\{x, y\}$ but there is a sequence $\left(u_{n}\right)_{n \in \mathbb{N}} \subset M$ converging to $x$ and

$$
\lim _{n \rightarrow+\infty} \frac{d\left(\pi_{x, y}\left(u_{n}\right), u_{n}\right)}{d\left(\pi_{x, y}\left(u_{n}\right), x\right)}=0 .
$$

Let $f \in S_{\operatorname{Lip}_{0}(M)}$ be such that $\frac{f(x)-f(y)}{d(x, y)}=1$. Let $\tilde{f}$ be a 1-Lipschitz extension of $f$ to $[x, y]$. Then

$$
\begin{aligned}
\left|f(x)-f\left(u_{n}\right)\right| & \geq\left|f(x)-\widetilde{f}\left(\pi_{x y}\left(u_{n}\right)\right)\right|-\left|\widetilde{f}\left(\pi_{x y}\left(u_{n}\right)\right)-f\left(u_{n}\right)\right| \\
& =d\left(x, \pi_{x y}\left(u_{n}\right)\right)-\left|\widetilde{f}\left(\pi_{x y}\left(u_{n}\right)\right)-f\left(u_{n}\right)\right| \\
& \geq d\left(x, \pi_{x y}\left(u_{n}\right)\right)-d\left(\pi_{x y}\left(u_{n}\right), u_{n}\right) \\
& \geq d\left(x, u_{n}\right)-2 d\left(\pi_{x y}\left(u_{n}\right), u_{n}\right)
\end{aligned}
$$

It follows that

$$
\lim _{n \rightarrow+\infty} \frac{\left|f(x)-f\left(u_{n}\right)\right|}{d\left(x, u_{n}\right)}=1
$$

and $f$ is not peaking at $(x, y)$.

(iii) $\Rightarrow$ (ii) Finally, since

$$
\frac{d\left(u, \pi_{x y}(u)\right)+d\left(v, \pi_{x y}(v)\right)}{d\left(\pi_{x y}(u), \pi_{x y}(v)\right)} \geq \min \left\{\frac{d\left(\pi_{x y}(u), u\right)}{d\left(\pi_{x y}(u), p\right)}, \frac{d\left(\pi_{x y}(v), v\right)}{d\left(\pi_{x y}(v), p\right)}\right\}
$$

we get

$$
\liminf _{u \rightarrow p} \frac{d\left(\pi_{x y}(u), u\right)}{d\left(\pi_{x y}(u), p\right)}=0
$$

if the liminf in (1) is 0 for some $p \in\{x, y\}$.

For the dual version of the proof we will need the following simple lemma which is valid in any metric space (see also [8]).

Lemma 3. Let $(M, d)$ be any metric space and suppose that $0 \in A \subset M$. If $f \in \operatorname{ext}\left(B_{\operatorname{Lip}_{0}(A)}\right)$, then $f_{S}, f_{I} \in \operatorname{ext}\left(B_{\operatorname{Lip}_{0}(M)}\right)$ where

$$
f_{S}(x):=\sup _{z \in A} f(z)-d(z, x) \quad \text { and } \quad f_{I}(x):=\inf _{z \in A} f(z)+d(z, x)
$$

for $x \in M$.

Note that $f_{S}$ resp. $f_{I}$ above are the smallest resp. the largest 1-Lipschitz extensions of $f$ (which basically gives the proof).

Proof. Let us give a proof for $f_{S}$. The proof for $f_{I}$ is similar. Clearly $f_{S}(x)=f(x)$ for $x \in A$ and $f_{S}$ is 1-Lipschitz as a supremum of 1-Lipschitz functions. Let $f_{S}=\frac{p+q}{2}, p, q \in B_{\operatorname{Lip}_{0} M}$. If $x \in A$, then $p(x)=q(x)=f(x)$ as $f \in \operatorname{ext}\left(B_{\operatorname{Lip}_{0} A}\right)$. If $x \in M \backslash A$, then $\forall z \in A$ :

$$
f(z)-p(x)=p(z)-p(x) \leq d(z, x) .
$$


Thus

$$
f_{S}(x)=\sup _{z \in A} f(z)-d(z, x) \leq p(x)
$$

By the same argument $f_{S}(x) \leq q(x)$. So $f_{S}(x)=p(x)=q(x)$ for all $x \in M$.

Theorem 4. Let $M$ be a complete subset of an $\mathbb{R}$-tree. If there is $b \in \operatorname{Br}(M) \backslash M$ then

a) there exist $\mu \neq \nu \in \operatorname{ext}\left(B_{\mathcal{F}(M)}\right)$ such that $\|\mu-\nu\|<2$.

b) there exist $f \neq g \in \operatorname{ext}\left(B_{\operatorname{Lip}_{0}(M)}\right)$ such that $\|f-g\|_{L}<2$.

Since the Lipschitz-free space over the completion of $M$ equals the Lipschitz-free space of $M$, this completness hypothesis is not restrictive.

Proof. a) Let the points $x^{\prime}, y^{\prime}, z^{\prime} \in M$ witness that $b \in \operatorname{Br}(M)$. For $p^{\prime} \in\left\{x^{\prime}, y^{\prime}, z^{\prime}\right\}$ we denote $\left.\left.M_{p^{\prime}}=\left\{w \in M: \pi_{b p^{\prime}}(w) \in\right] b, p^{\prime}\right]\right\}$. Then $M_{p^{\prime}}$ is closed in $M$ as $\pi_{b p^{\prime}}$ is continuous and $b$ is isolated from $M$. Notice that $p \in M_{p^{\prime}}$ satisfies (2) if there is $\alpha>0$ such that $d\left(w, \pi_{b p}(w)\right) \geq \alpha d\left(p, \pi_{b p}(w)\right)$ for all $w \in M_{p^{\prime}}$. We will show that for every $0<\alpha<1$ such a point $p$ exists. Indeed let $\frac{1-\alpha}{1+\alpha}=: \beta>0$ and set $f(w):=d(b, w)$. Then the Ekeland's variational principle [6] ensures the existence of a point $p \in M_{p^{\prime}}$ such that $f(p) \leq f(w)+\beta d(p, w)$ for all $w \in M_{p^{\prime}}$. It follows that

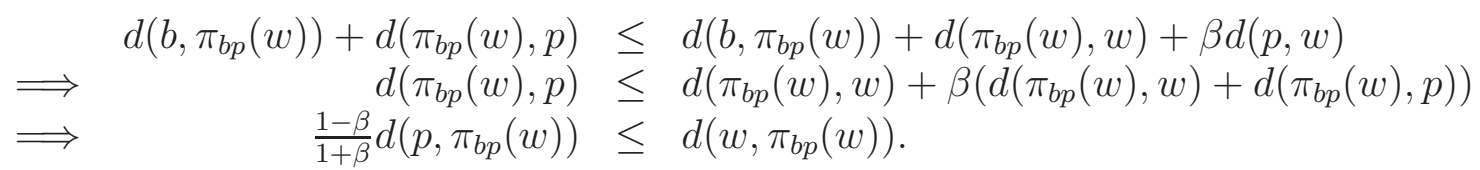

Thus, we see that we can find $x, y, z \in M$ such that (iii) in Proposition 2 is satisfied for the segments $[p, q]$ where $p \neq q \in\{x, y, z\}$. Proposition 1 then yields that $\frac{\delta_{p}-\delta_{q}}{d(p, q)}$ is an extreme point of the unit ball of $\mathcal{F}(M)$. Assuming, as we may, that $d(x, z) \leq d(z, y) \leq d(x, y)$, we obtain

$$
\begin{aligned}
\left\|\frac{\delta_{x}-\delta_{y}}{d(x, y)}-\frac{\delta_{z}-\delta_{y}}{d(y, z)}\right\|_{\mathcal{F}(M)} & =\left\|\frac{1}{d(x, y)}\left[\left(\delta_{x}-\delta_{z}\right)+\left(\delta_{z}-\delta_{y}\right)\right]-\frac{\delta_{z}-\delta_{y}}{d(y, z)}\right\|_{\mathcal{F}(M)} \\
& =\left\|\left[\frac{1}{d(x, y)}-\frac{1}{d(y, z)}\right]\left(\delta_{z}-\delta_{y}\right)+\frac{\delta_{x}-\delta_{z}}{d(x, y)}\right\|_{\mathcal{F}(M)} \\
& \leq d(z, y)\left[\frac{1}{d(y, z)}-\frac{1}{d(x, y)}\right]+\frac{d(x, z)}{d(x, y)} \\
& =1+\frac{d(x, z)-d(z, y)}{d(x, y)} \leq 1 .
\end{aligned}
$$

In conclusion, $\mu:=\frac{\delta_{x}-\delta_{y}}{d(x, y)}$ and $\nu:=\frac{\delta_{z}-\delta_{y}}{d(y, z)}$ are two extreme points of the unit ball of $\mathcal{F}(M)$ at distance less than or equal to 1.

b) We denote $\delta:=\inf \{d(w, b): w \in M\}$. Let $x, y, z$ be 3 points witnessing the fact that $b$ is a branching point. Two pointed metric spaces which differ only by the choice of the base point have isometric free spaces. This trivial observation allows us to assume that $x=0$ and that, for a fixed $0<\varepsilon<1$, we have $d(b, z)<(1+\varepsilon) \delta$. Let $M_{z}=\left\{w \in M: \pi_{z b}(w) \in(b, z]\right\}$. Let us consider the closed nonempty set $F=\left\{w \in M_{z}: d(b, z) \leq(1+\varepsilon) \delta\right\}$. Given $0<\alpha<1$ and using the Ekeland's variational principle as above, we may assume that $z$ satisfies $d\left(w, \pi_{z b}(w)\right) \geq$ $\alpha d\left(z, \pi_{z b}(w)\right)$ for all $w \in F$. Clearly $d\left(w, \pi_{z b}(w)\right) \geq \alpha d\left(z, \pi_{z b}(w)\right)$ for all $w \in M_{z} \backslash F$. 
We define $f(\cdot):=d(0, \cdot)$ on $M$ and then $g_{2}(\cdot):=d(0, \cdot)$ on $M \backslash M_{z}, g_{1}:=\left(g_{2}\right)_{S}$ on $\left(M \backslash M_{z}\right) \cup$ $\{z\}$ and finally $g:=\left(g_{1}\right)_{I}$ on $M$. Both $f, g \in \operatorname{ext}\left(B_{\operatorname{Lip}_{0}(M)}\right)$ by Lemma 3. The fact that $M$ is a subset of an $\mathbb{R}$-tree helps to write $g$ explicitely:

$$
g(w)= \begin{cases}d(0, w), & w \in M \backslash M_{z}, \\ d(0, b)-d(b, z)+d(z, w), & w \in M_{z} .\end{cases}
$$

It follows that $f(w)-g(w)=0$ for $w \in M \backslash M_{z}$ and $f(w)-g(w)=2 d\left(b, \pi_{z b}(w)\right)$ otherwise. We have

$$
\begin{aligned}
\|f-g\|_{L} & =\max \left\{\sup _{w_{1} \in M_{z}, w_{2} \notin M_{z}} \frac{2 d\left(b, \pi_{z b}\left(w_{1}\right)\right)}{d\left(w_{1}, w_{2}\right)}, \sup _{w_{1}, w_{2} \in M_{z}} \frac{2\left|d\left(w_{1}, \pi_{z b}\left(w_{1}\right)\right)-d\left(w_{2}, \pi_{z b}\left(w_{2}\right)\right)\right|}{d\left(w_{1}, w_{2}\right)}\right\} \\
& \leq \max \left\{\frac{2(1+\varepsilon) \delta}{2 \delta}, \frac{2}{1+\alpha}\right\}<2
\end{aligned}
$$

Theorem 5. Let $(M, d)$ be a complete metric space. The Lipschitz-free space over $M$ is isometric to $\ell_{1}(\Gamma)$ if and only if $M$ is of density $|\Gamma|$ and is negligible subset of an $\mathbb{R}$-tree $T$ which contains all the branching points of $T$.

Proof. The sufficiency follows from [9, Theorem 3.2]. Conversely, let us assume that $\mathcal{F}(M) \equiv$ $\ell_{1}(\Gamma)$. Then $M$ is of density $|\Gamma|$ and it must be 0 -hyperbolic by [9, Theorem 4.2]. In this case $T=\operatorname{conv}(M)$. If $\lambda_{T}(M)>0$, there is a set $A \subset[0,1]$ of positive measure such that $A$ embeds isometrically into $M$. Then $L_{1} \simeq \mathcal{F}(A) \subset \mathcal{F}(M) \equiv \ell_{1}(\Gamma)$ which is absurd. Since the extreme points of the ball (resp. dual ball) and their distances are preserved by bijective isometries we get by Theorem 5 a) (resp. b)) that $\operatorname{Br}(M) \subset M$.

Corollary 6. Let $M$ be an ultrametric space of cardinality at least 3. Then $\mathcal{F}(M)$ is not isometric to $\ell_{1}(\Gamma)$ for any $\Gamma$.

Proof. The completion of $M$ stays clearly ultrametric. Thus it can be isometrically embedded into an $\mathbb{R}$-tree [4]. However ultrametric spaces do not contain the interior of any segment, much less branching points.

\section{ISOMETRIES With SUBSPACES OF $\ell_{1}$}

We can now ask whether $\mathcal{F}(M)$ is isometric to a subspace of $\ell_{1}$ ? We are going to answer this question in the affirmative in the case when $M$ is supposed to be compact.

Lemma 7. Let $M$ be a compact subset an $\mathbb{R}$-tree such that $\lambda(M)=0$. Then $\lambda_{\operatorname{conv}(M)}(\overline{B r(M)})=$ 0 where the closure is taken in $\operatorname{conv}(M)$.

Proof. Clearly $\lambda_{\operatorname{conv}(M)}(\overline{B r(M)} \cap M)=0$. Assume that $\lambda_{\operatorname{conv}(M)}(\overline{B r(M)} \backslash M)>0$. Then $\overline{\operatorname{Br}(M)} \backslash M$ is uncountable. Hence there is some $\delta>0$ such that $\overline{\operatorname{Br}(M)} \cap\{x \in T: \operatorname{dist}(x, M) \geq \delta\}$ is uncountable and thus the set $\operatorname{Br}(M) \cap\left\{x \in T: \operatorname{dist}(x, M) \geq \frac{\delta}{2}\right\}$ is infinite. We conclude that there is an infinite $\delta$-separated family in $M$. This is absurd as $M$ was supposed to be compact. 
Proposition 8. Let $M$ be a compact subset of an $\mathbb{R}$-tree such that $\lambda(M)=0$. Then $\mathcal{F}(M)$ is isometric to a subspace of $\ell_{1}$.

Proof. Since $M$ is compact, $\operatorname{conv}(M)$ is compact and thus separable. Indeed, the mapping $\Phi: M \times M \times[0,1] \rightarrow \operatorname{conv}(M)$ defined by $\Phi(x, y, t):=\phi_{x y}(t d(x, y))$ is continuous by [3, Theorem II.4.1]. Now

$$
\mathcal{F}(M) \subseteq \mathcal{F}(B r(M) \cup M) \equiv \ell_{1}
$$

by [9, Corollary 3.4] as $\lambda_{\operatorname{conv}(M)}(\overline{B r(M) \cup M})=0$ by the previous lemma.

We do not know if the above proposition is valid when $M$ is supposed to be proper.

\section{Banach-Mazur distance to $\ell_{1}^{n}$}

In the case of finite subsets of $\mathbb{R}$-trees we get the following quantitative result.

Proposition 9. Let $M=\left\{x_{0}, x_{1}, \ldots, x_{n}\right\}, n \geq 2$, be a subset of a $\mathbb{R}$-tree. Let $x_{0}=0$ be the distinguished point. Let us suppose that

$$
0<\operatorname{sep}(M):=\frac{1}{2} \inf \{d(x, y)+d(x, z)-d(y, z): x, y, z \in M \text { distinct }\} .
$$

Then

$$
d_{B M}\left(\mathcal{F}(M), \ell_{1}^{n}\right)>\left(1-\frac{\operatorname{sep}(M)}{4 \operatorname{diam}(M)}\right)^{-1} .
$$

The condition $\operatorname{sep}(M)>0$ implies immediately that for each $x \neq y \in M$ we have $[x, y] \cap M=$ $\{x, y\}$. For the proof we will need the following lemmas. The first one is inspired by $[2$, Lemma 2.3].

Lemma 10. Let $X$ be a Banach space. Let $C=\bigcap_{i=1}^{n} x_{i}^{*-1}(-\infty, 1)$ where $x_{i}^{*} \in X^{*}$. Let $A \subset X \backslash C$ have the following property: for every $x \neq y \in A$, we have $\frac{x+y}{2} \in C$. Then the cardinality $|A|$ of $A$ is at most $n$.

Proof. For $x \in A$ let $\varphi(x):=i$ for some $i \in\{1, \ldots, n\}$ such that $x_{i}^{*}(x) \geq 1$. Since $1>$ $x_{\varphi(x)}^{*}\left(\frac{x+y}{2}\right)$ it follows that $x_{\varphi(x)}^{*}(y)<1$ for every $y \in A, y \neq x$. Thus $\varphi$ is injective and the claim follows.

Lemma 11. Let $f_{1}, \ldots, f_{2 n+1} \in S_{Y}$ such that $\left\|\frac{f_{i}+f_{j}}{2}\right\| \leq 1-\varepsilon$ for some $\varepsilon>0$ and all $1 \leq i \neq$ $j \leq 2 n+1$. Then $d_{B M}\left(Y, \ell_{\infty}^{n}\right)>(1-\varepsilon)^{-1}$.

Proof. Let $T: Y \rightarrow \ell_{\infty}^{n}$ such that $\|f\| \leq\|T f\|_{\infty} \leq(1+\varepsilon)\|f\|$. Then $\left\|T f_{i}\right\| \geq 1,\left\|\frac{T f_{i}+T f_{j}}{2}\right\|<1$ which is in contradiction with the previous lemma as $B_{\ell_{\infty}^{n}}^{O}$ is the intersection of $2 n$ halfspaces.

Proof of Proposition 9. Given $0 \leq i \neq j \leq n$, we will denote $\pi_{i j}:=\pi_{x_{i} x_{j}}$ the metric projection onto $\left[x_{i}, y_{j}\right]$. Further we define the function $f_{i j}: M \rightarrow \mathbb{R}$ as $f_{i j}(z):=d\left(x_{j}, \pi_{i j}(z)\right)$ for $z \in M$. Observe that since $\operatorname{sep}(M)>0$, this is the function peaking at $\left(x_{i}, x_{j}\right)$ from the proof of 
Proposition 2. It is clear that $\left|\frac{f_{i j}(x)-f_{i j}(y)}{d(x, y)}\right|=1$ if and only if $\{x, y\}=\left\{x_{i}, x_{j}\right\}$. We further have that

$$
\left|\frac{f_{i j}(x)-f_{i j}(y)}{d(x, y)}\right| \leq \frac{d(x, y)-\operatorname{sep}(M)}{d(x, y)} \leq 1-\frac{\operatorname{sep}(M)}{\operatorname{diam} M}
$$

for any other couple $x \neq y \in M$. Hence $\left\|\frac{f_{i j}+f_{k l}}{2}\right\|_{L} \leq 1-\frac{\operatorname{sep}(M)}{2 \operatorname{diam} M}$ for each $(i, j) \neq(k, l)$. Since $n \geq 2$, we have that $(n+1) n \geq 2 n+1$ and the result follows by Lemma 11 .

Remark 12. Note that the lower bound given in Proposition 9 is not optimal. This can be seen when $M=\left\{0, x_{1}, x_{2}\right\}$ is equilateral. We also don't know if this result extends to infinite subsets of $\mathbb{R}$-trees.

\section{REFERENCES}

[1] M. Bačák. Convex analysis and optimization in Hadamard spaces, De Gruyter, 2014.

[2] J. Borwein, J. Vanderwerff. Constructible convex sets, Set-Valued Anal. 12 (2004), no. 1, 61-77.

[3] M.R. Bridson and A. Haefliger, Metric spaces of non-positive curvature, Springer, 1999.

[4] P. Buneman. A note on the metric properties of trees, J. Combinatorial Theory Ser. B. 17 (1974) 48-50.

[5] M. Cúth and M. Doucha. Lipschitz-free spaces over ultrametric spaces, Mediterr. J. Math. (2015) DOI 10.1007/s00009-015-0566-7

[6] I. Ekeland. Nonconvex minimization problems, Bull. Amer. Math. Soc. 1 (1979), no. 3, 443-474

[7] S. N. Evans. Probability and Real Trees, LNM 1920, Springer, 2008.

[8] J.D. Farmer. Extreme points of the unit ball of the space of Lipschitz functions. Proc. Amer. Math. Soc. 121 (1994), no 3, 807-813.

[9] A. Godard, Tree metrics and their Lipschitz-free spaces, Proc. Amer. Math. Soc. 138 (2010), no. 12, 43114320.

[10] G. Godefroy and N.J. Kalton. Lipschitz-free Banach spaces. Studia Math. 159 (2003), no. 1, 121-141.

[11] N. Weaver. Lipschitz algebras. World Scientific Publishing Co. Inc., River Edge, NJ, 1999.

† Université Franche-Comté, Laboratoire de Mathématiques UMR 6623, 16 Route de Gray, 25030 Besançon Cedex, France

¥ Universidade Federal de São Paulo, Instituto de Ciência e Tecnologia, Campus São José dos Campos - Parque Tecnológico, Avenida Doutor Altino Bondensan, 500, 12247-016 São José Dos CAMPos/SP, BRAZIL

E-mail address: aude.dalet@univ-fcomte.fr

E-mail address: plkaufmann@unifesp.br

E-mail address: antonin.prochazka@univ-fcomte.fr 